\title{
Deep vein thrombosis in Sri Lankans - time to take notice
}

In 1984 Sheriffdeen (1) reported 16 cases of deep vein thrombosis (DVT) complicating vaginal hysterectomy, abdominal hysterectomy, myomectomy, caesarian section and sapheno-femoral ligation. He emphasised that the prevalence of DVT in the Sri Lankan population was appreciable, but probably an under-estimate, and suggested that raising awareness, and the use of Doppler ultrasound flow detector and venography, would increase the degree of diagnostic accuracy of DVT (1). Seventeen years later, the Doppler ultrasound flow detector is still not freely available in the state health service and no meaningful efforts have been made to prevent DVT or minimise the morbidity and mortality associated with it. This attitude may well have its roots in the false belief that DVT is rare among Sri Lankans and all Asians.

The use of ${ }^{125}$ I labelled fibrinogen alerted the western world to the reality that incidence of DVT in post-surgical patients in the UK was as high as $44 \%$ (2). Using the same technique, Shead and Narayanan in South India reported an incidence of $28 \%$ (3). Reports from Malaysia (4), Sudan (5), Hong Kong (6) and Thailand (7) give the respective incidence as $11.7 \%, 12.0 \%, 2.6 \%$ and $3.0 \%$. A literature survey shows that different researchers report varying results, even within the same country, on the incidence of DVT. It was stated in a standard textbook of surgery in 1984 (8), that "postoperative thrombosis is virtually unknown in tropical countries like Singapore". But in 1993, a group from this country reported an incidence of $11.4 \%$ after rectal surgery. Of these, three patients developed pulmonary embolism and one died (9). A Sri Lankan study in 1986 did not detect a single case of post-operative DVT using ${ }^{125}$ I fibrinogen in patients after prostatectomy (10). The University Surgical Unit of the Faculty of Medicine, Colombo, detected post-operative DVT in 63 of 137 consecutive patients $(45.9 \%)$ referred to the unit with a swollen leg over a 10 -month period. Of these patients $33 \%$ had a recent history of surgery or trauma (11). DVT also occurs world-wide in patients hospitalised for medical illnesses and pregnancy (12).

The major issues raised by DVT for clinicians include its incidence, methods for detection, prevention, management and predisposing factors. Although venography is the gold standard for the detection of venous thrombosis, it has to be carried out in a radiology department, the contrast is nephrotoxic, the test cannot be repeated too often (13), and in our experience, has a high incidence of complications at the injection site. In research studies ${ }^{125}$ I labelled fibrinogen is given pre-operatively and the leg is scanned with a rate meter daily thereafter for 7 to 10 days. It cannot be used in routine clinical practice (14). In impedance plethysmography a pneumatic cuff applied to the thigh is inflated to $50 \mathrm{mmHg}$, and the volume changes in the calf on inflation and deflation are measured using a plethysmograph. This equipment is expensive, sensitive to calibration and insensitive to calf vein thrombosis. Dislodging an embolus during the procedure is a dangerous complication (15).
The Doppler flow detector is a cheap, portable and user-friendly instrument. It will detect blood flow in the femoral vein and phasic flow during deep respiration, indicating patency of the inferior vena cava and iliac veins. An enhanced sound of increased flow with gentle thigh and calf compression also indicates patency. It is sufficiently accurate when compared with clinical assessment and ${ }^{129}$ I fibrinogen uptake (16). It has a false positive result if there are collaterals or patent superficial veins in the vicinity. In our experience, this procedure has a sensitivity of $70 \%$ and a specificity of $82.5 \%$. It is a useful bedside tool for screening purposes.

In real-time compression ultrasound scan the crosssectional compressibility of the deep veins is determined and it has been shown to be of value in determining their patency (15). Duplex (Triplex) ultrasound colour flow mapping is non-invasive and easily carried out. It is now emerging as the investigation of choice. The vein is easily identified by colour flow and its compressibility can be studied. Enhancement by distal momentary compression could be visualised. Moreover, by switching to pulsed wave Doppler signals, the phasic nature of flow, which confirms pelvic vein patency, could also be determined (17). In our experience it has a specificity of $94.8 \%$ and a sensitivity of $100 \%$.

\section{Prophylaxis}

The methods available for prophylaxis include elastic stockings (the simplest form of prophylaxis) used widely in West Germany (18), intermittent calf compression used by two-thirds of surgeons in the USA and by $30 \%$ in the UK (18), calf stimulation by electrical impulses used mostly in Switzerland (18), intravenous dextrans which have been studied in several trials (18), oral warfarin sodium used commonly by orthopaedic surgeons, and heparin, both low molecular weight (LMWH) and unfractionated (UH), used by most surgeons in the developed world. American surgeons tend to commence heparin prophylaxis after surgery, but European surgeons do so just before (18).

Kakkar and Lorenzo (19) identify four risk category groups for DVT.

i) Extremely low risk, under 40 years with no additional risk factors, over 40 years but undergoing minor surgery.

ii) Low risk, over 40 years having major abdominal surgery but no other risk factors.

ii) Moderate risk, major surgery with significant risk factors, (eg. obesity, varicose veins, malignancy or sepsis).

iv) High risk, over 40 years, either undergoing major hip or leg surgery, or those with other high risk factors (eg. malignancy, past history of DVT, coagulation disorders).

For low risk patients there is no indication for prophylaxis (20). All others should have graduated compression 
stockings or mechanical compression of veins intermittently during surgery. Heparin prophylaxis is a must for both high and moderate risk category patients. The indications for prophylaxis in the low risk category are best worked out on an individual basis even for procedures such as varicose vein surgery (21). A study in the UK has determined that over $10 \%$ of post-operative deaths in hospital are due to fatal pulmonary embolism (19). In the light of overwhelming evidence for thromboprophylaxis, it is extremely difficult to understand why prophylactic measures have not been introduced in Sri Lankan operating theatres and wards as a policy.

Guidelines for a hospital thromboprophylaxis policy should include the following.

1 Provision of graduated compression stockings and pneumatic intermittent calf compression devices to be used on all patients over the age of 40 years.

2 A uniform heparin policy of LMWH 2500 to 5000 units 2 hours before surgery, and 2500 daily for 5 to 7 days (or until full mobilisation), in individuals deemed at risk.

3 High risk patients should be given 5000 units of UH subcutaneously 2 hours pre-operatively and 5000 units 12 hourly. LMWH 5000 units 2 hours pre-operatively, followed by 5000 units daily is an alternate regime (19). Heparin should be continued until the patient is fully ambulant.

In a Sri Lankan setting it would be more convenient and cheaper to use the freely available UH. Intra-operative blood loss and haematoma formation following heparin prophylaxis in the post-operative period is always an alarming one for the surgeon. However, well conducted trials have shown that the benefits of thromboprophylaxis outweigh the risks.

\section{Treatment}

In the majority of patients DVT starts in the calf. The clots remain confined to the calf in about $80 \%$ of patients, and do not extend into the main popliteal veins (22). These patients do not develop pulmonary embolism as a rule.

In the remaining $20 \%$, the clot may extend into the popliteal and femoral veins, and there is danger of embo$\operatorname{lism}(23)$.

Graduated compression stockings, early ambulation and low dose aspirin were at one time the main therapeutic regimes for early DVT in the calf with no evidence of propagation. Clinical trials, however, have shown that this category too needs active treatment to prevent the sequel to DVT ie. deep venous insufficiency. The simultaneous use of intravenous heparin and oral warfarin therapy has become standard clinical practice for all patients with venous thromboembolism who are medically stable (24). Our practice in the University Surgical Unit is to commence treatment with a bolus dose of 5000 units of UH followed by an infusion of 15000 units of heparin in $500 \mathrm{ml}$ of isotonic saline over every 12 hours. The dose of heparin is adjusted by monitoring the activated partial thromboplastin time (APTT) 4 to 6 hours after commencement of therapy and daily thereafter, to keep it at $1 \frac{1}{2}$ times the normal value. Oral warfarin sodium is commenced in a dose of $10 \mathrm{mg}$ given immediately, followed by $5 \mathrm{mg}$ daily. The infusion of heparin is continued until the patient has no pain and the oedema subsides. The dose of oral warfarin sodium is adjusted by determining the International Normalised Ratio (INR) from the prothrombin time. It is kept between 2 and 3. Asymptomatic patients need heparin only until the INR is satisfactory. The patient is then ambulated, preferably with stockings, and advised to continue the warfarin sodium orally for 3 months following femoro-popliteal DVT, or for 6 months in the case of ilio-femoral DVT.

LMWHs have recently been developed for clinical use. Compared with UH, these preparations have a longer plasma life. Because of their pharmacokinetic properties a stable and sustained anticoagulant effect is obtained when it is administered once or twice daily. Besides, laboratory monitoring is not essential (25).

There is controversy over the routine use of thrombolytic agents such as streptokinase, urokinase and recombinant tissue plasminogen activator (26). The advantage in using these drugs is that they reduce the incidence of deep venous insufficiency following recanalisation, but their side effects and cost preclude routine use.

Surgical thrombectomy is rarely practiced as a routine procedure for DVT. Vena caval obstruction early in the course of DVT is one indication for surgical thrombectomy. Massive DVT with phlegmasia cerulea dolens or venous gangrene is an absolute indication for surgery. Fasciotomy, thrombectomy, placement of a Greenfield filter, and construction of a temporary arterio-venous fistula to maintain high venous flow rates that prevent thrombosis in the thrombectomised vein, may save life and limb (27).

Greenfield filters are introduced percutaneously either via the internal jugular vein, or the femoral vein if patent, using radiological imaging. The filter is placed in the infrarenal inferior vena cava. The specific indication for this procedure is repeated pulmonary embolism. Prophylactic use is also indicated in individual cases where the risk of pulmonary embolism is estimated to be high. The cost of a filter with the accessories is about Rs. 150 000 .

Venous thromboembolism has become an important cause of morbidity and mortality. In the USA 100000 to 200 000 deaths are attributed annually to venous thromboembolism (28). Patients over the age of 40 years undergoing surgery are at the highest risk. Patients after myocardial infarction, stroke and complicated pregnancies are nonsurgical groups at risk. The clinical diagnosis of DVT is inaccurate. Most fatalities in pulmonary embolism occur within 30 minutes of the embolic episode (29). Hence prophylaxis against thromboembolism becomes important in clinical practice. It is time to appreciate these dangers and the need for prophylaxis. It is also time for specialists in all disciplines to form consensus groups and to set down practical guidelines for the routine use of thromboprophylaxis in high risk groups. Surgeons should initiate this process, as DVT is more common in surgical practice. 


\section{References}

1. Sheriffdeen AH. Vascular emergencies. Sri Lankan Family Physician 1981; 8: 131-49.

2. Kakkar V, Howe CT, Nicolaides AN, Renney JTG, Clarke MB. Deep vein thrombosis of the leg. American Journal of Surgery 1970; 120: 527-30.

3. Shead GV, Narayanan R. Incidence of postoperative venous thromboembolism in South India. British Journal of Surgery 1980; 67: 813-4.

4. Cunningham IGE, Yong NK. The incidence of postoperative deep vein thrombosis in Malaysia. British Journal of Surgery 1974; 61: 482-3.

5. Hassan MA, Rahman EA, Rahman IA. Post-operative deep vein thrombosis in Sudanese patients. British Medical Journal 1973; 1: 515-7.

6. Nandi P, Wong KP, Wei WI, Ngan H, Ong GB. Incidence of post-operative deep vein thrombosis in Hong Kong Chinese. British Journal of Surgery 1980; 67: 251-3.

7. Chumnijarakij T, Poshyachinda V. Post-operative thrombosis in Thai women. Lancet 1975; 21: 1357-8.

8. Harding Rains AJ, David Ritchie RH (eds). Bailey and Love's Short Practice of Surgery. London: ELBS / H Lewis, 1984.

9. Cum CK, Sim EK, Ngoi SS. Deep vein thrombosis complicating colorectal surgery in the Chinese in Singapore. Annals of the Academy of Medicine Singapore 1993; 22: 895-7.

10. Sarath Kumara KA, Ratnasena BJN, Herath KB, Ratnatunga PCA. The incidence of postoperative deep vein thrombosis in Sri Lankan patients. Proceedings of the Annual Scientific Sessions of the College of Surgeons of Sri Lanka, 1986.

11. Wijeratne SM, Sheriffdeen AH. Proceedings of Sri Lanka Medical Association 112th Anniversary Academic Sessions 1999: 23.

12. Greer IA. Thrombosis in pregnancy; maternal and fetal issues. Lancet 1999; 353: 1258-65.

13. Lensing AW, Prandoni $P$, Buller HR. Contrast venography: results and side effects. Radiology 1993; 177: 503-9.

14. Comerota AJ, Katz ML, Grossi RJ. The comparative value of non-invasive testing for diagnosis and surveillance of deep vein thrombosis. Journal of Vascular Surgery 1988; 7: 40-9.

15. Heijboer J, Buller HG, Lensing AW. A comparison of real time ultrasonography with impedence plethysmography for the diagnosis of deep vein thrombosis in symptomatic out patients. New England Journal of Medicine 1993; 329: 1365-9.
16. Williams OB, McCaffrey JF, Lau OJ. Deep vein thrombosis in a Queensland Hospital. British Medical Journal 1973; 1: 517-9.

17. Bradley MJ, Spencer PA, Alexander L, Milner GR. Colour flow mapping in the diagnosis of the calf deep vein thrombosis. Clinical Radiology 1993; 47: 399-402.

18. Cooke ED. The current practice of prevention. In: Bergqvist D, Comerata AJ, Nicolaides AN, Scurr JH (eds). Prevention of venous thromboembolism. London: Med-Orion, 1994: 123 -9.

19. Kakkar VV, de Lorenzo F. Prevention of venous thromboembolism in general surgery. In: Pineo GF, Hull RD (eds). Clinical Haematology. Prevention. Diagnosis and Management of Venous Thromboembolic disease. London: Balliere Tindall, 1998: 675-87.

20. Riber C, Alstrusp N. Post-operative thromboembolism after day case herniorrhaphy. British Journal of Surgery 1996; 83: 420-1.

21. Streeter EH, Qureshi T, Harvey MR, Corbett CRR. Thromboembolism after varicose vein surgery. British Joumal of Surgery 1998; 81: (suppl 1): 26.

22. Nicolaides AV, Kakkar VV, Field ES et al. Venous stasis and deep vein thrombosis. British Journal of Surgery 1972; 59: 51-3.

23. Kakkar VV, Howe CT, Flanc C, Clarke MB. Natural history of deep vein thrombosis. Lancet 1969; 2: $230-4$.

24. Gallus A, Jackman J, Tillett J, Safety and efficacy of warfarin started early after submassive venous thrombosis or pulmonary embolism. Lancet 1986; 2: 1293-6.

25. Bratt G, Tornebotim E, Widland L, Lockner D. Low molecular weight heparin: Pharmacokinetics after intravenous and subcutaneous administration in human volunteers. Thrombosis Research 1986; 42: 613-20.

26. Gallus AS. Thrombolytic therapy for venous thrombosis and pulmonary embolism. In: Pineo GF, Hull RD (eds). Clinical Haematology. Prevention, Diagnosis and Management of Venous Thromboembolic disease. London: Balliere Tindal, 1998: 663-73.

27. Bo Eklof. The value of thrombectomy, In: Bergqvist $D$, Comerota AJ. Nicolaides AN, Scurr JH (eds). Prevention of venous thromboembolism. London: Med-Orion, 1994: 357. 71.

28. Kumar R, Mc Kinney WP, Raj G. Perioperative prophylaxis of venous thromboembolism. American Journal of Medical Science 1993; 306: 336-44.

A H Sheriffdeen, Professor and Head, M Wijeyaratne, Senior Lecturer, Department of Surgery, Faculty of Medicine, University of Colombo. 